\title{
Quantitative STEM-EDS Mapping and Analysis
}

\author{
Chad M. Parish and Luke N. Brewer
}

Sandia National Laboratories, PO Box 5800 MS 1411, Albuquerque, NM, USA, 87185

Modern analytical electron microscopes, operated in spectrum imaging (SI) modes, allow qualitative and quantitative mapping of materials' chemistry below $10 \mathrm{~nm}$ in spatial resolution [1,2]. Such a capability is useful to understand and interpret properties of advanced materials, such as $(\mathrm{Pb}, \mathrm{La})(\mathrm{Zr}, \mathrm{Ti}) \mathrm{O}_{3}$ "PLZT" dielectrics, which suffer degraded properties when the cations are inhomogenously distributed. Figure 1 illustrates cation ratio maps of sintered PLZT measured using a published STEM-EDS technique [2]. STEM-EDS high-resolution maps have low count rates. Multivariate statistical analysis (MVSA) methods de-noise the individual pixels of STEM-EDS SIs, improving signal-to-noise in resultant quantitative maps [3]. Although MVSA reduces the noise in the SI, it is unclear precisely how the noise structure is changed via MVSA. Despite the apparent reduction in experimental scatter, the data in any pixel is based on a small number of counts and the analyst must view the results critically. Quantification involves not just measurement of the chemical content, but also analysis of the uncertainty of that measurement. In order to correctly interpret quantified SIs, it is necessary to know how error and uncertainty propagate through the acquisition, processing, and quantification steps. We have used Monte Carlo simulations to study how uncertainty propagates through SI processing for quantitative STEM-EDS mapping.

Poisson photon-counting statistics are commonly applied to study uncertainty in non-SI EDS data [4]. We used Monte Carlo simulations of SIs to compare actual SI-to-SI scatter vs. standard deviations calculated from Poisson statistics. Figure 2a illustrates the starting point for a Monte Carlo simulation consisting of 32 nominally identical $64 \times 64$ pixel SIs, each with random Poisson noise added at a mean level of 1,000 counts per pixel. Figures $2 \mathrm{~b}-\mathrm{c}$ show the MVSA+spatial simplicity [5] result for one of the 32 slices, extracting two distinct phases (perovskite and fluorite PLZT). From these scores (Figure 2b) and loadings (Figure 2c), the cation fractions are calculated at each pixel [2]. The standard deviations at that pixel, such as $\Delta(\mathrm{Zr} / \mathrm{Ti})$, can be calculated using Poisson-based methods from the integrated counts for the $\mathrm{Zr}$ and Ti peaks [4]. Profiles based on such Poisson-analyses are shown as black solid lines in Figure 2e. Figure 2d shows how the MVSAresultant mean and standard deviations are found from the repeated simulations, and these are given as blue dashed lines in Figure 2e. It is seen in Figure 2e that standard deviations calculated from Poisson statistics are larger than the SI-to-SI standard deviation. We infer that traditional Poissonstatistics do not describe the actual pixel-to-pixel variation in MVSA-processed quantified spectrum images. Thus, we conclude that error propagation and noise level in quantified SIs may not be amenable to Poisson statistics. Other methods of analysis may be more appropriate to the question of quantification of STEM-EDS SIs. Additional experiments and Monte Carlo simulations are underway and may provide insight into error and uncertainty in quantification of EDS SIs.

[1] Watanabe et al., Micros. Microanal. 12 (2006) P.515.

[2] Parish et al., J. Am. Ceram. Soc. 91 (2008) P.3690

[3] Burke et al., J. Mat. Sci. 41 (2006) P.4512.

[4] Williams \& Carter, Transmission Electron Microscopy, Plenum, New York (1996).

[5] Keenan, Surface and Interface Analysis 41 (2009) P.79. 
Sandia is a multiprogram laboratory operated by Sandia Corporation, a LockheedMartin Company, for the United States Department of Energy's National Nuclear Security Administration under contract DE-AC04-94AL85000.
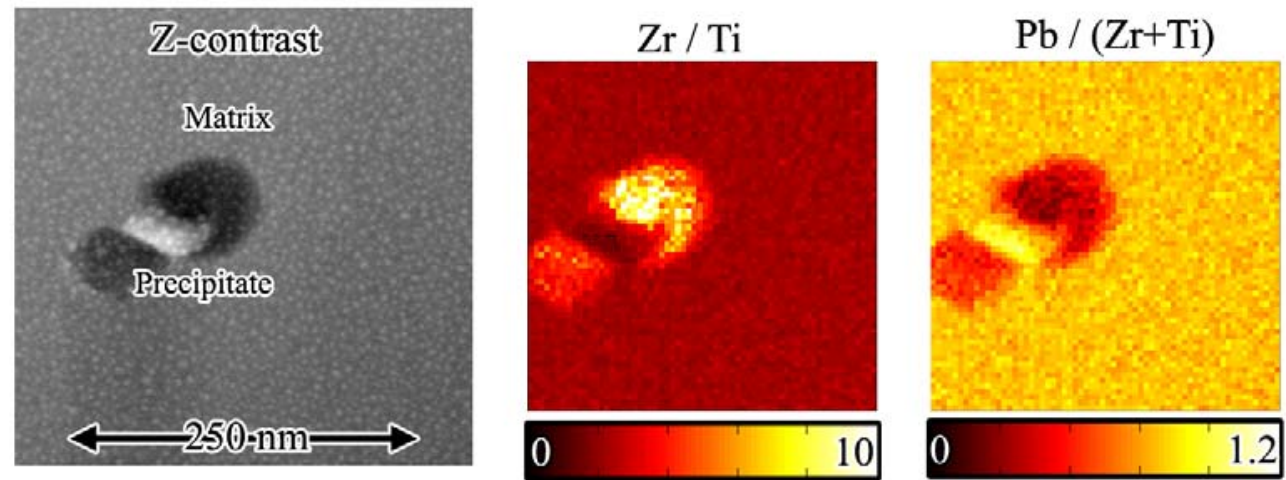

Figure 1: Z-contrast image and quantitative STEM-EDS maps of a precipitate in a matrix of nominal $\left(\mathrm{Pb}_{0.88} \mathrm{La}_{0.12}\right)\left(\mathrm{Zr}_{0.68} \mathrm{Ti}_{0.29}\right) \mathrm{O}_{3}$ PLZT. Quantitative values are cation molar fraction ratios.

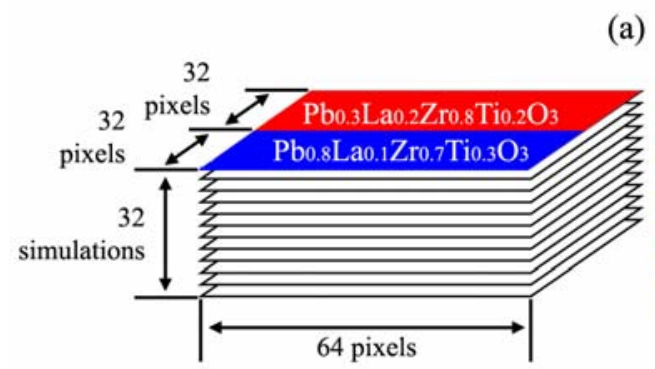

(a)

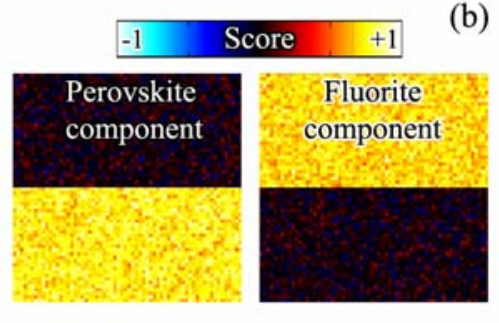

(b)

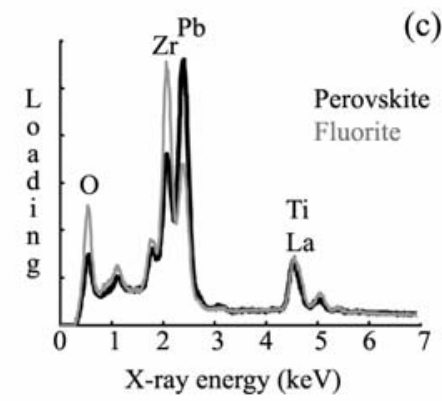

(c)
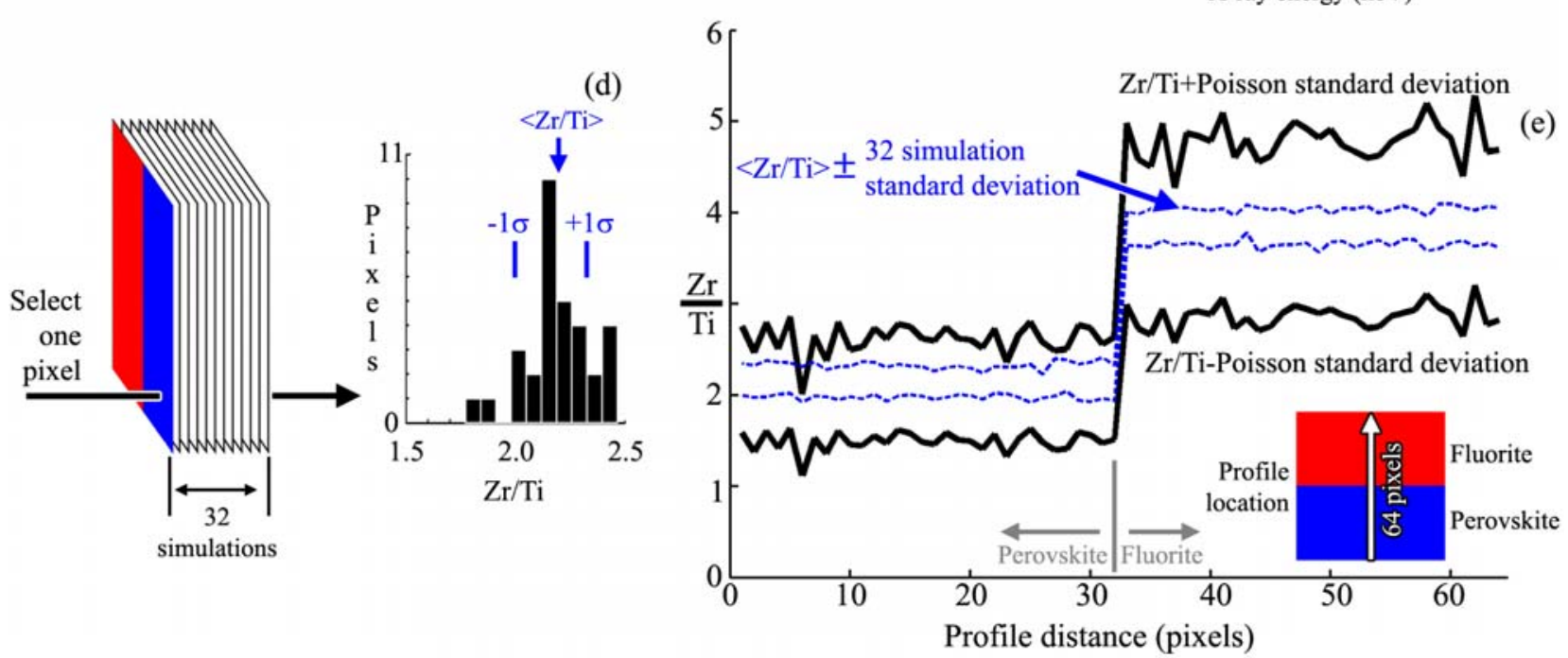

Figure 2: (a) Schematic illustration of simulations, performed with 1,000 counts/pixel Poisson noise. (b-c) Spatial-simplicity results for one slice of the simulation. (d) Schematic illustration of extracting the post-MVSA mean and standard deviation of a quantification parameter at any pixel. (e) Profiles of Poisson-based and MVSA-based quantifications \pm standard deviations. 